\title{
The study of myocardial protective effect of creatine phosphate sodium in liver transplant patients
}

\author{
Xiaoguang Yuan ,'Xiaguang Duan, Yuqing Kang \\ The Third Affiliated Hospital of Inner Mongolia Medical University, Hohhot, China
}

Received: March 1, 2015

DOI: $10.14725 /$ dcc.v2n2p8
Accepted: April 2, $2015 \quad$ Online Published: June 15, 2015

URL: http://dx.doi.org/10.14725/dcc.v2n2p8

\begin{abstract}
Objective: To study the cardioprotective effectof creatine phosphate sodium by measuring perioperative myocardial enzymes of allogeneic liver transplant patients.

Methods: The 26 cases of orthotopic liver transplant patients were randomly divided into control group and creatine phosphate sodium group, 13 cases in each group. The creatine phosphate sodium group were given creatine phosphate sodium $30 \mathrm{mg} / \mathrm{kg}$ at the time of induction. The control group were given equal amount of N.S. All patients at six-phase of before surgery, after anesthesia,before anhepatic phase, anhepatic phase,new liver and the 12 hours after surgery extraction radial artery. The CK, CK-MB and cTnI activity was measured.

Results: To compared with control group, creatine phosphate sodium group's plasma CK, CK-MB and cTnI concentrations in before anhepatic phase, anhepatic phase, new liver and the 12 hours after surgery 4 phase are significantly lower $(p<0.05)$.

Conclusions: The creatine phosphate sodium has a protective effect on liver transplantation in patients with myocardial damage.
\end{abstract}

Key Words: Creatine Phosphate Sodium, Liver transplantation, Myocardial protection

Researchers reach a consensus ${ }^{[1]}$ that there is a close pathologic correlation between heart and liver in patients underwent liver transplantation. (1) simultaneous damage to liver, heart and other organs by alcohol; (2) liver cirrhosis secondary to heart disease; (3) effects of primary heart disease on liver transplantation; (4) cardiac injury by liver transplantation. It has been 50 years since the clinical application of liver transplantation when the liver transplantation was completed by Starzl, the founder of liver transplantation, in 1963. Though a great progress has been made in the field of medical community, liver transplantation remains the only effective treatment for end-stage liver disease. This paper aims to explore the myocardial protective effect of creatine phosphate sodium during perioperative period, and its suitable medication timing.

\section{Clinical data and methods}

\subsection{General information}

Since 2005, 26 cases of liver transplantation in our hospital were performed in male patients, aging from 32 to 48 years old, body weight $62-78 \mathrm{~kg}$, ASA grade from II to III, 17 cases of hepatitis B cirrhosis, and 9 cases of liver cancer. Orthotopic liver transplantation was applied in our case. Preoperative electrocardiogram (ECG) and serum creatine kinase (CK), creatine kinase isoenzyme MB (CK-MB), cardiac troponin I (cTnI) concentration and electrolyte examination results were found to be normal. The 26 cases of orthotopic liver transplant patients were randomly divided into

\footnotetext{
*Correspondence: Xiaoguang Yuan; E-mail: bgyyyxg@163.com; Address: The Third Affiliated Hospital of Inner Mongolia Medical University, Hohhot, China
} 
control group and creatine phosphate sodium (CPS) group, with 13 cases in each group.

\subsection{Methods of Anesthesia}

After the patient entering into the operating room, electrocardiogram, non-invasive blood pressure and pulse oxygen saturation were closely monitored, and radial artery cannula were placed under local anesthesia for continuous monitoring of arterial blood pressure. Preoperative medication: intramuscular injection of Atropine $0.5 \mathrm{mg}$ half an hour before operation. intravenous-inhalation combined anesthesia induction: intravenous injection of midazolam $0.05 \mathrm{mg} / \mathrm{kg}$, fentanyl 4-6 g/kg, propofol 1.5-2 $\mathrm{mg} / \mathrm{kg}$, and Vecuronium $0.1-0.15 \mathrm{mg} / \mathrm{kg}$. Internal jugular vein puncture was performed and three deep vein puncture needles were placed, using propofol TCI + sevoflurane 1mac for anesthesia, and discontinuous additional administration of fentanyl and vecuronium was applied. Continuous infusion of dopamine ( $3 \mathrm{mg} / \mathrm{kg} / \mathrm{min}$ ), and ulinastatin was taken until the end of operation. Adjust the respiratory parameters to maintain the end tidal carbon dioxide partial pressure in the normal range during inhalation of air and oxygen. During induction, intravenous injection of creatine phosphate sodium $30 \mathrm{mg} / \mathrm{kg}$ was implemented in CPS group, and equivalent amount of normal saline was injected in control group. Adjust the infusion speed and application of vasoactive drugs according to BP and CVP. Adjust the stability of internal environment according to blood gas, electrolyte, biochemical results and input - output volume. Supplementary of crystal liquid, plasma, red blood cell and albumin was needed and rational use of vasoactive drugs and volume therapy is essential in the light of blood routine, coagulation function results. Timely replenishment of the electrolyte to regulate blood sugar to correct acid-base imbalance was vital, and heating mattress as well as transfusion heating device was used for temperature regulation.

\subsection{Monitoring index}

ECG, pulse oximetry, nasopharyngeal temperature (T), arterial blood pressure (systolic blood pressure, diastolic blood pressure SBP DBP), central venous pressure (CVP), end tidal carbon dioxide were monitored during the surgery. Sample blood from radial artery was taken and plasma CK, CK-MB and cTnI activity were measured at 6 phases, that is, presurgery, after anesthesia, preanhepatic, anhepatic and neohepatic period, and 12 hours after the surgery.

\subsection{Statistical methods}

The results were analyzed by SPSS software according to statistics method, and all the data were shown in the form of mean plus or minus standard deviation $( \pm s)$. Statistical analysis was performed using repeated measures analysis of variance, and difference was statistically significant when $p<0.05$.

Table 1: Changes of plasma CK, CK-MB and cTnI concentrations in the two groups during perioperative period $(\bar{x} \pm s)$

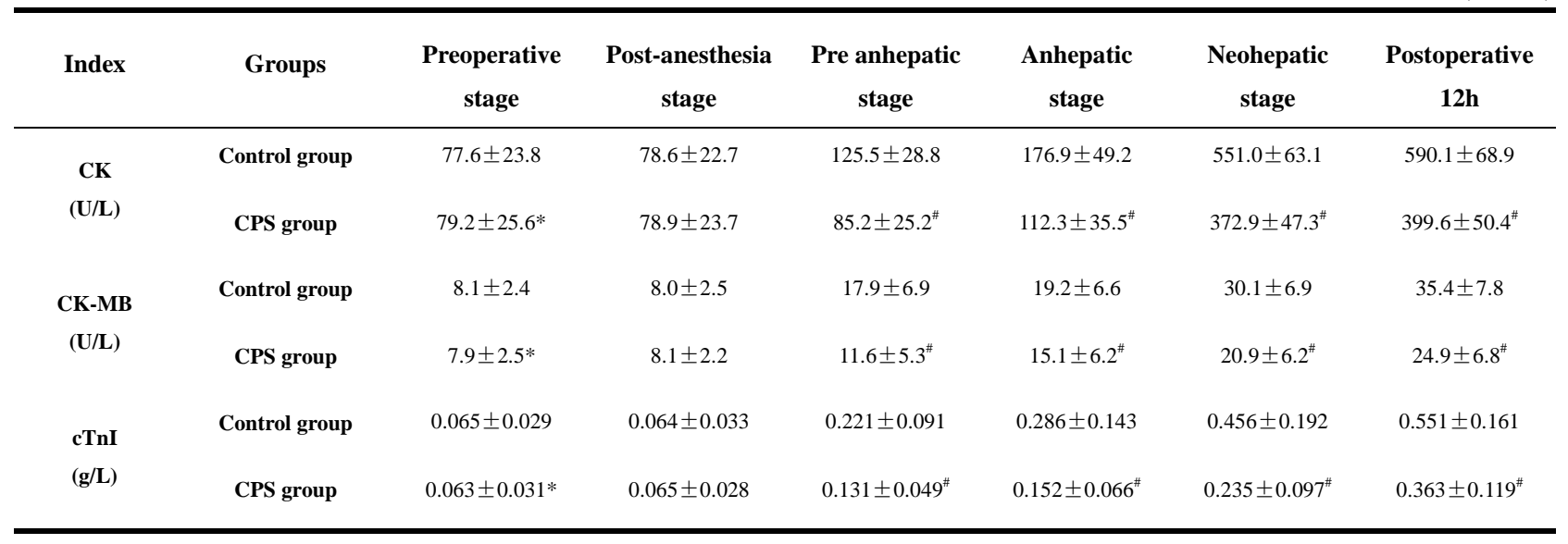

Note.*p $>0.05 ; \quad{ }^{*} p<0.05$

\section{Results}

There was no significant difference in gender, age, operation time, blood loss, and CK, CK-MB and cTnI concentrations between the two groups ( $p>0.05)$. There was no significant difference in plasma CK, CK-MB and cTnI concentrations between the two groups after anaesthesia $(p>0.05)$. CK,
CK-MB and cTnI concentration during anhepatic phase in two groups gradually increased until postoperative 12 hours. Compared with the control group, the levels of CK, CKMB and cTnI concentration during preanhepatic, anhepatic, neohepatic period, and postoperative 12 hours of four phase were significantly decreased $(p<0.05)$ (see Table 1$)$. 


\section{Discussion}

Many researchers investigate the pathogenesis of postoperative cardiovascular disease to date by retrospectively studying cardiovascular complications after liver transplantation. ${ }^{[2-4]}$ The mechanism of perioperative myocardial injury after liver transplantation is very complex, including primary end-stage liver disease, ${ }^{[5]}$ intraoperative ischemia, ${ }^{[6]}$ liquid supplement, ${ }^{[7]}$ acidic metabolites and endotoxin in large quantities to enter the systemic circulation. ${ }^{[8]}$

Long time occlusion of the inferior vena cava during liver transplantation can reduce the amount of cardiac output and decrease the oxygen supply, which can lead to low cardiac output and blood pressure reduction and myocardial ischemic injury. Myocardial ischemia and hypoxia may cause blood vessels in the brain stem to constrict and stimulate central nervous system through carotid chemoreceptor. Moreover, sympathetic efferent fiber was enhanced, and secretion of endogenous catecholamine increase, which resulted in ventricular arrhythmia. Myocardial ischemia and hypoxia is prone to induce arrhythmias due to electrical instability. The transition from aerobic to anaerobic metabolism could occur rapidly in response to myocardial ischemia and hypoxia, leading to a decrease in the generation of ATP, as well as the decrease of the high-energy phosphate content in myocardial cells, especially to phosphocreatine. Long time ischemia can lead to depletion of phosphocreatine during the operation. Perioperative anesthesia, surgical trauma and other complex factors may aggravate coronary artery insufficiency, finally resulting in dysfunction of autonomic nervous system. ${ }^{[3]}$ Incidence of adverse cardiac events and adverse events increased greatly. The main clinical manifestations include: sinus tachycardia tachycardia, atrial premature beat, room premature beat, ventricular tachycardia tachycardia, ventricular tachycardia tachycardia, atrial fibrillation. Early myocardial injury after liver transplantation is associated with adverse cardiovascular events.

When the permeability of damaged myocardial cell membrane increased, a large number of myocardial cells like CK, CK-MB, would enter blood stream. Therefore, CK-MB and $\mathrm{CK}$ can serve as markers of myocardial injury, for the diagnosis of myocardial injury. Troponin C (TNC), troponin $\mathrm{T}$ (TNT) and cardiac troponin I (TNI), the three subunits of troponin, are important regulatory proteins of human striated muscle contraction. TnI is inhibitory subunit of an actin. With the in-depth study of cTnI, cTnI is considered to be the best marker for the diagnosis of myocardial damage, with regard to the specificity of the myocardium or the diagnostic sensitivity.

In this study, CK, CK-MB and cTnI gradually increased at the beginning of the preanhepatic phase, and significantly rose at neohepatic stage phase, and they still stuck at a high level postoperative 12 hours. Therefore, it is concluded that different degree of myocardial injury occurred from pre anhepatic phase, new liver reperfusion period to early postoperative phase.

In this study, the levels of CK, CK-MB and cTnI concentration in pre anhepatic, anhepatic period, neohepatic stage and postoperative 12 hours of four phase were significantly decreased $(p<0.05)$ in CPS group. It indicates that creatine phosphate sodium has a protective effect on the heart so that myocardial damage is lighter. Strumia E et al ${ }^{[9,10]}$ also have shown that creatine phosphate sodium could protect heart.

Our study on myocardial protection of creatine phosphate sodium and other researches were compared, and found that advanced administration of CPS carried better treatment effect during anesthesia induction phase than that at anhepatic and neohepatic phase (peak period of myocardial injury). The positive relationship of myocardial injury protection with the level and reserve quantity of creatine phosphate in vivo could be employed to explain the phenomenon.

Myocardial protection function of CPS is possibly associated with the following effects. Stabilize sarcolemma by inhibiting nucleotide enzyme decomposition and keeping intracellular adenine nucleotide levels, inhibition of phospholipid degradation of myocardial ischemia area, and through inhibition of ADP induced platelet aggregation to improve microcirculation in ischemic area. CPS is an efficient energy supply material, and it contains creatine molecule, a vital energy for cells and chemical energy reserve for myocardium and skeletal muscle. When a sufficient amount of CPS is injected, ADP would transform to ATP under the effect of CPK and other isoenzymes, which is directly uptook and utilized by myocardial cells to improve cardiac function. In addition, promote the red blood cells to tissue uptake of sufficient oxygen, ensure energy supplies with $\mathrm{Na}^{+}$ - $\mathrm{K}^{+}$- ATP ion pump, $\mathrm{Ca}^{2+}$ pump to increase cell membrane stability, and reduce the occurrence of arrhythmia. Moreover, it can inhibit the accumulation of the hemolytic esterase, protect myocardial cells phospholipid against free radical damage, thereby protect myocardial cells.

Sodium phosphocreatine imposes protective effect on myocardium in patients undergoing liver transplantation, however causes to myocardial damage in liver transplantation patients are complex and diverse, which requires reasonable prevention and treatment according to the etiology. Further research and exploration of sodium phosphocreatine on myocardial protection mechanism and medication time and dosage are essential.

\section{Conflicts of Interest Disclosure}

The authors have no conflict of interest related to this article. 


\section{References}

[1] Ripoll C, Yotti R, Bermejo J, Banares R. The heart in liver transplantation [J]. J Hepatol, 2011, 54(4): 810-22.

[2] Zamirian M, Tavassoli M, Aghasadeghi K. Corrected QT interval and QT dispersion in cirrhotic patients before and after liver transplantation [J]. Arch Iran Med, 2012, 15(6): 375-7.

[3] Huh IY, Kim YK, Shin WJ, Park SE, Bang JY, Hwang GS. Increased B-type natriuretic peptide during liver transplantation: relationship to invasively measured hemodynamic parameters [J]. Transplant Proc, 2012, 44(5): 1318-22.

[4] Lentine KL, Costa SP, Weir MR, et al. Cardiac disease evaluation and management among kidney and liver transplantation candidates: a scientific statement from the American Heart Association and the American College of Cardiology Foundation [J]. J Am Coll Cardiol, 2012, 60(5): 434-80.

[5] Xu ZD, Xu HT, Yuan HB, et al. Postreperfusion syndrome during orthotopic liver transplantation: a single-center experience [J]. Hep- atobiliary Pancreat Dis Int, 2012, 11(1): 34-9.

[6] Leithead JA, Tariciotti L, Gunson B, et al. Donation after cardiac death liver transplant recipients have an increased frequency of acute kidney injury [J]. Am J Transplant, 2012, 12(4): 965-75.

[7] Uemura T, Ramprasad V, Hollenbeak CS, Bezinover D, Kadry Z. Liver transplantation for hepatitis $\mathrm{C}$ from donation after cardiac death donors: an analysis of OPTN/UNOS data [J]. Am J Transplant, 2012, 12(4): 984-91.

[8] Giakoustidis A, Cherian TP, Antoniadis N, Giakoustidis D. Combined cardiac surgery and liver transplantation: three decades of worldwide results [J]. J Gastrointestin Liver Dis, 2011, 20(4): 41521.

[9] Strumia E, Pelliccia F, D'Ambrosio G. Creatine phosphate: pharmacological and clinical perspectives [J]. Adv Ther, 2012, 29(2): 99-123.

[10] Miao P, Sun B, Feng X. Treatment of myocardial damage with creatine phosphate following neonatal asphyxia: a meta-analysis $[\mathrm{J}]$. Chinese Journal of Contemporary Pediatrics, 2012, 14(3): 172-6. 